\title{
Erratum to: Ultra high energy photons and neutrinos with JEM-EUSO
}

\section{The JEM-EUSO Collaboration}

Published online: 24 July 2015

(C) Springer Science+Business Media Dordrecht 2015

\section{Erratum to: Exp Astron}

DOI 10.1007/s10686-013-9353-2

The original publication of this article (10.1007/s10686-013-9353-2) unfortunately contained an error. The collaboration name "The JEM-EUSO Collaboration" was not inserted in the authorgroup.

The full collaboration's author list is provided here which was missing in the original version.

\section{The JEM-EUSO Collaboration}

J.H. Adams Jr. ${ }^{m d}$, S. Ahmad ${ }^{b b}$, J.-N. Albert ${ }^{b a}$, D. Allard ${ }^{b c}$, L. Anchordoqui ${ }^{m f}$, V. Andreev ${ }^{m e}$, A. Anzalone ${ }^{d h, d n}$, Y. Arai ${ }^{e v}$, K. Asano ${ }^{e t}$, M. Ave Pernas ${ }^{k c}$, P. Baragatti ${ }^{d o}$, P. Barrillon ${ }^{b a}$, T. Batsch ${ }^{h c}$, J. Bayer ${ }^{c d}$, R. Bechini ${ }^{d l}$, T. Belenguer $^{k b}$, R. Bellotti ${ }^{d a, d b}$, K. Belov ${ }^{m e}$, A.A. Berlind ${ }^{m h}$, M. Bertaina ${ }^{d k, d l}$, P.L. Biermann ${ }^{c b}$, S. Biktemerova ${ }^{i a}$, C. Blaksley ${ }^{b c}$, N. Blanc ${ }^{l a}$, J. Błe, cki $^{\text {hd }}$, S. Blin-Bondil ${ }^{b b}$, J. Bl"umer ${ }^{c b}$, P. Bobik ${ }^{j a}$, M. Bogomilov ${ }^{a a}$, M. Bonamente ${ }^{m d}$, M.S. Briggs ${ }^{m d}$, S. Briz ${ }^{k d}$, A. Bruno ${ }^{d a}$, F. Cafagna ${ }^{d a}$, D. Campana ${ }^{d f}$, J-N. Capdevielle ${ }^{b c}$, R. Caruso ${ }^{d c, d n}$, M. Casolino ${ }^{e w, d i}$, C. Cassardo ${ }^{d k, d l}$, G. Castellini ${ }^{d d}$, C. Catalano ${ }^{b d}$, O. Catalano ${ }^{d h, d n}$, A. Cellino ${ }^{d k, d m}$, M. Chikawa ${ }^{e d}$, M.J. Christl ${ }^{m g}$, D. Cline ${ }^{m e}$, V. Connaughton ${ }^{m d}$, L. Contido ${ }^{\text {do G. Cordero }}{ }^{\text {ga }}$, H.J. Crawford ${ }^{m a}$, R. Cremonini ${ }^{d l}$, S. Csorna ${ }^{m h}$, S. Dagoret-Campagne ${ }^{b a}$, A.J. de Castro ${ }^{k d}$, C. De Donato ${ }^{d i}$, C. de la Taille ${ }^{b b}$, C. De Santis ${ }^{d i, d j}$, L. del Peral ${ }^{k c}$, A. Dell'Oro ${ }^{d k, d m}$, N. De Simone ${ }^{d i}$, M. Di Martino ${ }^{d k, d m}$, G. Distratis ${ }^{c d}$, F. Dulucq ${ }^{b b}$, M. Dupieux ${ }^{b d}$, A. Ebersoldt ${ }^{c b}$, T. Ebisuzaki ${ }^{e w}$, R. Engel ${ }^{c b}$, S. Falk ${ }^{c b}$, K. Fang ${ }^{m b}$, F. Fenu ${ }^{c d}$, I. Fernández-Gómez ${ }^{k d}$, S. Ferrarese ${ }^{d k, d l}$, D. Finco ${ }^{d o}$, M. Flamini ${ }^{d o}$, C. Fornaro ${ }^{d o}$, A. Franceschid ${ }^{d e}$, J. Fujimoto ${ }^{e v}$,

The online version of the original article can be found at http://dx.doi.org/10.1007/s10686-013-9353-2.

A. D. Supanitsky dalesupa@gmail.com

G. Medina-Tanco gmtanco@nucleares.unam.mx

\section{A. Guzmán} guzman@astro.uni-tuebingen.de

1 Instituto de Astronomía y Física del Espacio (IAFE), CONICET-UBA, Buenos Aires, Argentina

2 Instituto de Ciencias Nucleares, UNAM, A. P. 70-543, 04510 México, DF, México

3 Institute for Astronomy and Astrophysics, Kepler Center, University of Tubingen, Tubingen, Germany 
M. Fukushima ${ }^{e g}$, P. Galeotti ${ }^{d k, d l}$, G. Garipov ${ }^{i c}$, J. Geary ${ }^{m d}$, G. Gelmini ${ }^{m e}$, G. Giraudo ${ }^{d k}$, M. Gonchar ${ }^{i a}$, C. González Alvarado ${ }^{k b}$, P. Gorodetzky ${ }^{b c}$, F. Guarino ${ }^{d f, d g}$, A. Guzmán ${ }^{c d}$, Y. Hachisu ${ }^{e w}$, B. Harlov ${ }^{i b}$, A. Haungs ${ }^{c b}$, J. Hernández Carretero ${ }^{k c}$, K. Higashide ${ }^{e r e w}$, D. Ikeda ${ }^{e g}$, H. Ikeda ${ }^{e p}$, N. Inoue ${ }^{e r}$, S. Inoue ${ }^{e g}$, A. Insolia ${ }^{d c, d n}$, F. Isgr'o ${ }^{d f, d p}$, Y. Itow ${ }^{e n}$, E. Joven ${ }^{k e}$, E.G. Judd ${ }^{m a}$, A. Jung ${ }^{f b}$, F. Kajino ${ }^{e i}$, T. Kajino ${ }^{e l}$, I. Kaneko ${ }^{e w}$, Y. Karadzhov ${ }^{a a}$, J. Karczmarczyk ${ }^{h c}$, M. Karus ${ }^{c b}$, K. Katahira ${ }^{e w}$, K. Kawai ${ }^{e w}$, Y. Kawasaki ${ }^{e w}$, B. Keilhauer ${ }^{c b}$, B.A. Khrenov ${ }^{i c}$, Jeong-Sook Kim ${ }^{f a}$, Soon-Wook Kim ${ }^{f a}$, Sug-Whan Kim ${ }^{f d}$, M. Kleifges ${ }^{c b}$, P.A. Klimov ${ }^{i c}$, D. Kolev ${ }^{a a}$, I. Kreykenbohm ${ }^{c a}$, K. Kudela ${ }^{j a}$, Y. Kurihara ${ }^{e v}$, A. Kusenko ${ }^{m e}$, E. Kuznetsov ${ }^{m d}$, M. Lacombe ${ }^{b d}$, C. Lachaud ${ }^{b c}$, J. Lee ${ }^{f c}$, J. Licandro ${ }^{k e}$, H. Lim ${ }^{f c}$, F. López ${ }^{k d}$, M.C. Maccarone ${ }^{d h, d n}$, K. Mannheim ${ }^{c e}$, D. Maravilla ${ }^{g a}$, L. Marcelli ${ }^{d j}$, A. Marinide ${ }^{d e}$ O. Martinez ${ }^{g c}$, G. Masciantonio ${ }^{d i, d j}$, K. Mase ${ }^{e a}$, R. Matev ${ }^{a a}$, G. Medina-Tanco ${ }^{g a}$, T. Mernik ${ }^{c d}$, H. Miyamoto ${ }^{b a}$, Y. Miyazaki ${ }^{e c}$, Y. Mizumoto ${ }^{e l}$, G. Modestino ${ }^{d e}$, A. Monaco ${ }^{d a, d b}$, D. Monnier-Ragaigne ${ }^{b a}$, J.A. Morales de los Ríos ${ }^{k a, k c}$, C. Moretto ${ }^{b a}$, V.S. Morozenko ${ }^{i c}$, B. Mot $^{\text {bd }}$, T. Murakami ${ }^{e f}$, M. Nagano ${ }^{e c}$, M. Nagata ${ }^{e h}$, S. Nagataki ${ }^{e k}$, T. Nakamura ${ }^{e j}$, T. Napolitano ${ }^{d e}$, D. Naumov ${ }^{i a}$, R. Nava ${ }^{g a}$, A. Neronov ${ }^{l b}$, K. Nomoto $^{e u}$, T. Nonaka ${ }^{e g}$, T. Ogawa ${ }^{e w}$, S. Ogio ${ }^{e o}$, H. Ohmori ${ }^{e w}$, A.V. Olinto ${ }^{m b}$, P. Orleański ${ }^{h d}$, G. Osteria ${ }^{d f}$, M.I. Panasyuk ${ }^{i c}$, E. Parizot ${ }^{b c}$, I.H. Park ${ }^{f c}$, H.W. Park ${ }^{f c}$, B. Pastircak ${ }^{j a}$, T. Patzak ${ }^{b c}$, T. Paul ${ }^{m f}$, C. Pennypacker ${ }^{m a}$, S. Perez Cano ${ }^{k c}$, T. Peter ${ }^{l c}$, P. Picozza ${ }^{d i, d j, e w}$, T. Pierog $^{c b}$, L.W. Piotrowski ${ }^{e w}$, S. Piraino ${ }^{c d, d h}$, Z. Plebaniak ${ }^{h c}$, A. Pollinil ${ }^{l a}$, P. Prat ${ }^{b c}$, G. Prévôt ${ }^{b c}$, H. Prieto ${ }^{k c}$, M. Putis ${ }^{j a}$, P. Reardon ${ }^{m d}$, M. Reyes ${ }^{k e}$, M. Riccide, I. Rodríguez ${ }^{k d}$, M.D. Rodríguez Frías ${ }^{k c}$, F. Ronga ${ }^{d e}$, M. Roth ${ }^{c b}$, H. Rothkaehl ${ }^{h d}$, G. Roudil ${ }^{b d}$, I. Rusinov ${ }^{a a}$, M. Rybczyński ${ }^{h a}$, M.D. Sabau ${ }^{k b}$, G. Sáez Cano ${ }^{k c}$, H. Sagawa ${ }^{e g}$, A. Saito ${ }^{e j}$, N. Sakaki ${ }^{c b}$, M. Sakata ${ }^{e i}$, H. Salazar ${ }^{g c}$, S. Sánchez ${ }^{k d}$, A. Santangelo ${ }^{c d}$, L. Santiago Crúz ${ }^{g a}$, M. Sanz Palomino ${ }^{k b}$, O. Saprykin ${ }^{i b}$, F. Sarazin ${ }^{m c}$, H. Sato ${ }^{e i}$, M. Sato ${ }^{e s}$, T. Schanz ${ }^{c d}$, H. Schieler ${ }^{c b}$, V. Scotti ${ }^{d f, d g}$, A. Segreto ${ }^{d h, d n}$, S. Selmane ${ }^{b c}$, D. Semikoz ${ }^{b c}$, M. Serra ${ }^{k e}$, S. Sharakin ${ }^{i c}$, T. Shibata ${ }^{e q}$, H.M. Shimizu ${ }^{e m}$, K. Shinozaki ${ }^{e w, c d}$, T. Shirahama ${ }^{e r}$, G. Siemieniec-Ozie, bło ${ }^{h b}$, H.H. Silva López ${ }^{g a}$, J. Sledd ${ }^{m g}$, K. Słomińska ${ }^{h d}$, A. Sobey $^{m g}$, T. Sugiyama ${ }^{e m}$, D. Supanitsky ${ }^{g a}$, M. Suzuki ${ }^{e p}$, B. Szabelska ${ }^{h c}$, J. Szabelski ${ }^{h c}$, F. Tajima ${ }^{e e}$, N. Tajima ${ }^{e w}$, T. Tajima $^{c c}$, Y. Takahashi ${ }^{e s}$, H. Takami ${ }^{e v}$, M. Takeda ${ }^{e g}$, Y. Takizawa ${ }^{e w}$, C. Tenzer ${ }^{c d}$, O. Tibolla ${ }^{c e}$, L. Tkachev ${ }^{i a}$, H. Tokuno ${ }^{e t}$, T. Tomida ${ }^{e w}$, N. Tone ${ }^{e w}$, S. Toscano ${ }^{l b}$, F. Trillaud ${ }^{g a}$, R. Tsenov ${ }^{a a}$, Y. Tsunesada ${ }^{e t}$, K. Tsuno $^{e w}$, T. Tymieniecka ${ }^{h c}$, Y. Uchihori ${ }^{e b}$, M. Unger ${ }^{c b}$, O. Vaduvescu ${ }^{k e}$, J.F. Valdés-Galicia ${ }^{g a}$, P. Vallania ${ }^{d k, d m}$, L. Valore ${ }^{d f, d g}$, G. Vankova ${ }^{a a}$, C. Vigorito ${ }^{d k, d l}$, L. Villaseñor ${ }^{g b}$, P. von Ballmoos ${ }^{b d}$, S. Wada ${ }^{e w}$, J. Watanabe ${ }^{e l}$, S. Watanabe ${ }^{e s}$, J. Watts Jr. ${ }^{m d}$, M. Weber ${ }^{c b}$, T.J. Weiler ${ }^{m h}$, T. Wibig ${ }^{h c}$, L. Wiencke ${ }^{m c}$, M. Wille ${ }^{c a}$, J. Wilms ${ }^{c a}$, Z.Włodarczyk $^{h a}$, T. Yamamoto ${ }^{e i}$, Y. Yamamoto ${ }^{e i}$, J. Yang $^{f b}$, H. Yano ${ }^{e p}$, I.V. Yashin ${ }^{i c}$, D. Yonetoku ${ }^{e f}$, K. Yoshida $^{e i}$, S. Yoshida ${ }^{e a}$, R. Young ${ }^{m g}$, M.Yu. Zotov ${ }^{i c}$, A. Zuccaro Marchi ${ }^{e w}$

${ }^{a a}$ St. Kliment Ohridski University of Sofia, Bulgaria

${ }^{b a}$ LAL, Univ Paris-Sud, CNRS/IN2P3, Orsay, France

${ }^{b b}$ Omega, Ecole Polytechnique, CNRS/IN2P3, Palaiseau, France

${ }^{b c}$ APC, Univ Paris Diderot, CNRS/IN2P3, CEA/Irfu, Obs. de Paris, Sorbonne Paris Cité, France

${ }^{b d}$ IRAP, Université de Toulouse, CNRS, Toulouse, France

${ }^{c a}$ ECAP, University of Erlangen-Nuremberg, Germany

${ }^{c b}$ Karlsruhe Institute of Technology (KIT), Germany

${ }^{c c}$ Ludwig Maximilian University, Munich, Germany

${ }^{c d}$ Inst. for Astronomy and Astrophysics, Kepler Center, University of T"'ubingen, Germany

${ }^{c e}$ Institut f ur Theoretische Physik und Astrophysik, University of W"urzburg, Germany

${ }^{d a}$ Istituto Nazionale di Fisica Nucleare - Sezione di Bari, Italy

${ }^{d b}$ Universita' degli Studi di Bari Aldo Moro and INFN - Sezione di Bari, Italy

${ }^{d c}$ Dipartimento di Fisica e Astronomia - Universita' di Catania, Italy

${ }^{d d}$ Consiglio Nazionale delle Ricerche (CNR) - Ist. di Fisica Applicata Nello Carrara, Firenze, Italy

de Istituto Nazionale di Fisica Nucleare - Laboratori Nazionali di Frascati, Italy

$d f$ Istituto Nazionale di Fisica Nucleare - Sezione di Napoli, Italy

${ }^{d g}$ Universita' di Napoli Federico II - Dipartimento di Scienze Fisiche, Italy

${ }^{d h}$ INAF - Istituto di Astrofisica Spaziale e Fisica Cosmica di Palermo, Italy

${ }^{d i}$ Istituto Nazionale di Fisica Nucleare - Sezione di Roma Tor Vergata, Italy

${ }^{d j}$ Universita' di Roma Tor Vergata - Dipartimento di Fisica, Roma, Italy

${ }^{d k}$ Istituto Nazionale di Fisica Nucleare - Sezione di Torino, Italy

${ }^{d l}$ Dipartimento di Fisica, Universita' di Torino, Italy

${ }^{d m}$ Osservatorio Astrofisico di Torino, Istituto Nazionale di Astrofisica, Italy

${ }^{d n}$ Istituto Nazionale di Fisica Nucleare - Sezione di Catania, Italy

${ }^{d o}$ UTIU, Dipartimento di Ingegneria, Rome, Italy 
${ }^{d p}$ DIETI, Universita' degli Studi di Napoli Federico II, Napoli, Italy

${ }^{e a}$ Chiba University, Chiba, Japan

${ }^{e b}$ National Institute of Radiological Sciences, Chiba, Japan

${ }^{e c}$ Fukui University of Technology, Fukui, Japan

${ }^{e d}$ Kinki University, Higashi-Osaka, Japan

${ }^{e e}$ Hiroshima University, Hiroshima, Japan

${ }^{e f}$ Kanazawa University, Kanazawa, Japan

${ }^{e g}$ Institute for Cosmic Ray Research, University of Tokyo, Kashiwa, Japan

${ }^{e h}$ Kobe University, Kobe, Japan

${ }^{e i}$ Konan University, Kobe, Japan

${ }^{e j}$ Kyoto University, Kyoto, Japan

${ }^{e k}$ Yukawa Institute, Kyoto University, Kyoto, Japan

${ }^{e l}$ National Astronomical Observatory, Mitaka, Japan

${ }^{e m}$ Nagoya University, Nagoya, Japan

${ }^{e n}$ Solar-Terrestrial Environment Laboratory, Nagoya University, Nagoya, Japan

${ }^{e o}$ Graduate School of Science, Osaka City University, Japan

${ }^{e p}$ Institute of Space and Astronautical Science/JAXA, Sagamihara, Japan

${ }^{e q}$ Aoyama Gakuin University, Sagamihara, Japan

er Saitama University, Saitama, Japan

${ }^{e s}$ Hokkaido University, Sapporo, Japan

${ }^{e t}$ Interactive Research Center of Science, Tokyo Institute of Technology, Tokyo, Japan

${ }^{e u}$ University of Tokyo, Tokyo, Japan

${ }^{e v}$ High Energy Accelerator Research Organization (KEK), Tsukuba, Japan

${ }^{e w}$ RIKEN, Wako, Japan

${ }^{f a}$ Korea Astronomy and Space Science Institute (KASI), Daejeon, Republic of Korea

${ }^{f b}$ Ewha Womans University, Seoul, Republic of Korea

${ }^{f c}$ Sungkyunkwan University, Seoul, Republic of Korea

${ }^{f d}$ Center for Galaxy Evolution Research, Yonsei University, Seoul, Republic of Korea

${ }^{g a}$ Universidad Nacional Autónoma de México (UNAM), Mexico

${ }^{g b}$ Universidad Michoacana de San Nicolas de Hidalgo (UMSNH), Morelia, Mexico

${ }^{g c}$ Benemérita Universidad Autónoma de Puebla (BUAP), Mexico

${ }^{h a}$ Jan Kochanowski University, Institute of Physics, Kielce, Poland

${ }^{h b}$ Jagiellonian University, Astronomical Observatory, Krakow, Poland

${ }^{h c}$ National Centre for Nuclear Research, Lodz, Poland

${ }^{h d}$ Space Research Centre of the Polish Academy of Sciences (CBK), Warsaw, Poland

${ }^{i a}$ Joint Institute for Nuclear Research, Dubna, Russia

${ }^{i b}$ Central Research Institute of Machine Building, TsNIIMash, Korolev, Russia

${ }^{i c}$ Skobeltsyn Institute of Nuclear Physics, Lomonosov Moscow State University, Russia

${ }^{j a}$ Institute of Experimental Physics, Kosice, Slovakia

${ }^{k a}$ Consejo Superior de Investigaciones Científicas (CSIC), Madrid, Spain

${ }^{k b}$ Instituto Nacional de Técnica Aeroespacial (INTA), Madrid, Spain

${ }^{k c}$ Universidad de Alcalá (UAH), Madrid, Spain

${ }^{k d}$ Universidad Carlos III de Madrid, Spain

${ }^{k e}$ Instituto de Astrofísica de Canarias (IAC), Tenerife, Spain

${ }^{l a}$ Swiss Center for Electronics and Microtechnology (CSEM), Neuchatel, Switzerland

${ }^{l b}$ ISDC Data Centre for Astrophysics, Versoix, Switzerland

${ }^{l c}$ Institute for Atmospheric and Climate Science, ETH Z"urich, Switzerland

${ }^{m a}$ Space Science Laboratory, University of California, Berkeley, USA

${ }^{m b}$ University of Chicago, USA

${ }^{m c}$ Colorado School of Mines, Golden, USA

${ }^{m d}$ University of Alabama in Huntsville, Huntsville, USA

${ }^{m e}$ University of California (UCLA), Los Angeles, USA

${ }^{m f}$ University of Wisconsin-Milwaukee, Milwaukee, USA

${ }^{m g}$ NASA - Marshall Space Flight Center, USA

${ }^{m h}$ Vanderbilt University, Nashville, USA 